Itinéraires Itinéraires

Littérature, textes, cultures

2013-2 | 2014

Sade et les femmes

\title{
Sade, les femmes et le féminisme dans les années
} 1950

L'identité et le genre en question

\section{Perrine Coudurier}

\section{OpenEdition}

\section{Journals}

Édition électronique

URL : http://journals.openedition.org/itineraires/674

DOI : $10.4000 /$ itineraires.674

ISSN : 2427-920X

Éditeur

Pléiade

\section{Édition imprimée}

Date de publication : 1 mars 2014

Pagination : $59-71$

ISBN : 978-2-343-02712-8

ISSN : $2100-1340$

\section{Référence électronique}

Perrine Coudurier, « Sade, les femmes et le féminisme dans les années 1950 », Itinéraires [En ligne], 2013-2 | 2014, mis en ligne le 01 novembre 2013, consulté le 01 mai 2019. URL : http:// journals.openedition.org/itineraires/674 ; DOI : 10.4000/itineraires.674

\section{(c) (i) (2)}

Itinéraires est mis à disposition selon les termes de la licence Creative Commons Attribution - Pas d'Utilisation Commerciale - Pas de Modification 4.0 International. 


\title{
Sade, les femmes et le féminisme dans les années 1950
}

\author{
L’identité et le genre en question
}

\section{Résumé}

Et si la femme était le personnage principal chez Sade? C'est ce que suggère Jean Paulhan dans les années 1950, tant dans ses études sur Sade que dans sa préface au texte sadien Histoire d'O de Pauline Réage, dans laquelle il identifie l'auteur anonyme à Justine et fait d'O une dominatrice dans la soumission. L'influence de la conception de la femme par Sade est ainsi interrogée à travers la réception de l'œuvre par deux couples d'écrivains antithétiques: Paulhan et Réage d'une part, Sartre et Beauvoir d'autre part. Le Deuxième Sexe et Histoire d'O offrent en effet une relecture métaphorique de Sade, proposant aux lectrices de passer de Justine la naïve à Juliette la femme libre, en connaissance de cause. Sade permet ainsi de « redessiner l'individualité, de repenser la maîtrise et d'imaginer d'autres façons d'être » (Annie Lebrun, Ailleurs et autrement, Gallimard, 2011, p. 260); il anticipe paradoxalement la démythification de l'éternel féminin et les questionnements portant sur le genre.

Mots clés : Sade, Beauvoir, Réage, féminisme, genre

\section{Abstract}

This paper is a study of the relationships between Sade and his posterity in the Fifties, in France, around the representation of women being. Simone de Beauvoir and Pauline Réage are two authoresses who make Sade an important thinker of the female condition. Between fascination, submission and rebellion, they explain the difficult position of the women both in literature and in society. It seems to be difficult, for Sade and these two authoresses, to avoid a mythic image of women and to escape the binarity man/woman.

Keywords: Sade, Beauvoir, Réage, feminism, gender studies

Certaines coïncidences éditoriales sont symptomatiques pour l'histoire des idées. Ainsi, peut-on ignorer que Simone de Beauvoir publie en l'espace 
de cinq ans Le Deuxième Sexe ${ }^{1}$, Faut-il brûler Sade ${ }^{2}$ ? et Les Mandarins ${ }^{3}$, mais encore que cette dernière œuvre est contemporaine du livre le plus scandaleux des années 1950 : Histoire d'O de Pauline Réage ${ }^{4}$ ? Plus précisément, la recherche génétique a montré que Beauvoir lit Sade en 1949, l'année où elle rédige son essai féministe, tandis qu'elle écrit Les Mandarins en même temps que ce qui deviendra Privilèges. Enfin, notons que Réage défend les écrits de Beauvoir ${ }^{5}$. Au sein de ces relations et publications singulières, Sade est un des pivots de cette pensée féminine d'aprèsguerre, qui prône l'émancipation de la femme. Si le $\mathrm{xx}^{\mathrm{e}}$ siècle a pris Sade au sérieux, comme l'a démontré Éric Marty ${ }^{6}$, Beauvoir et Réage sont au cœur de cette rémanence du marquis.

La référence à Sade paraît singulière lorsque l'on traite de la libération de la femme, lui qui illustre de façon récurrente dans ses romans la domination et la violence masculines. Pourtant, c'est par rapport à cet auteur que les deux femmes se situent; elles adoptent une posture ambivalente oscillant entre continuation du projet sadien et rébellion à son encontre. Leurs œuvres, à la fois essais et fictions, dessinent un chemin entre Justine et Juliette, entre soumission et insoumission. Explorant les ambiguïtés du couple et les tensions entre masculin et féminin, elles redessinent une carte du Tendre inversée, où le mal est accepté et où la conception de l'égalité ne correspond à aucun canon. Au point de vue fantasmatique masculin de Sade dans ses romans, ces œuvres opposent un regard rationnel - Beauvoir et fantasmatiquement féminin - Réage - qui bouscule les représentations traditionnelles du couple.

Ainsi, notre approche tentera de déployer ces ambivalences et ces révolutions, en explorant l'influence ontologique et littéraire de Sade dans la redéfinition de la femme, pour montrer qu'il existe dans ces œuvres du $\mathrm{XVIII}^{\mathrm{e}}$ et du XXe siècle une troisième voie entre féminin et masculin, concourant à un « trouble dans le genre ${ }^{7}$ ». Sade, Beauvoir, Réage et le « troisième sexe » : le marquis interroge le genre et l'identité, et devient l'écho contemporain des questionnements d'après-guerre.

1. Simone de Beauvoir, Le Deuxième Sexe, Paris, Gallimard, 1949.

2. La première publication dans Les Temps modernes a lieu en 1951-1952, puis dans le recueil d'articles Privilèges, chez Gallimard, en 1955 et en 1972.

3. Simone de Beauvoir, Les Mandarins, Paris, Gallimard, 1954.

4. Pauline Réage, Histoire d'O, Paris, Pauvert, 1954, réédité en 1975.

5. Dominique Aury, dont Pauline Réage est le pseudonyme, écrira des articles sur

Le Deuxième Sexe, Les Mandarins puis La Force de l'âge dans la NRF.

6. Éric Marty, Pourquoi le XXe siècle a-t-il pris Sade au sérieux?, Paris, Seuil, 2011.

7. Judith Butler, Trouble dans le genre. Le féminisme et la subversion de l'identité [1990], Paris, La Découverte, 2006. 


\section{Et Sade créa la femme : une réception féminine de Sade}

Quels critères retenir pour analyser les deux approches singulières de Beauvoir et Réage? Une précision terminologique s'impose. Sade donne lieu à une postérité complexe, qui alterne entre dimension sadique et dimension sadienne. Éric Marty retrace la généalogie de cette descendance dans l'introduction de son essai Pourquoi le XX siècle a-t-il pris Sade au sérieux? Il marque le lien entre régime éditorial et sérieux des interprétations. Ainsi, au XIX ${ }^{\mathrm{e}}$ siècle, les œuvres du marquis n'ont pas été rééditées, et la réception est de l'ordre du sadisme; on considère Sade comme un écrivain lié au folklore du libertinage. Le $\mathrm{xx}^{\mathrm{e}}$ siècle, au contraire, grâce au travail éditorial de Maurice Heine dans les années 1930, tend à abandonner progressivement ces représentations caricaturales. Le siècle se scinde en deux moments de réception : un premier $\mathrm{xx}^{\mathrm{e}}$ siècle qui insiste sur la mythologie sadienne - Sade victime de tous les pouvoirs - tandis que 1947 marque un basculement. Sade est alors pris au sérieux, les analyses importantes se multiplient et c'est l'homme sadien qui captive, en dehors de l'aspect érotique :

\footnotetext{
C'est un Sade d'une ambition inouïe qui surgit. Ambition philosophique, ambition politique, ambition historique, ambition esthétique qu'on retrouve de manière parfois contradictoire, mais en fait structurellement très homogène, chez Adorno, Klossowski, Bataille et Blanchot. Sade permet alors d'interroger le surgissement du sujet pervers comme nouveau sujet de l'histoire ${ }^{8}$.
}

Notre travail se place dans la lignée des analyses d'Éric Marty, tout en les prolongeant sur deux points. Sur le plan de la périodisation littéraire tout d'abord: Beauvoir et Réage publient à la charnière du siècle, elles reflètent ainsi, selon nous, simultanément les deux pans distingués par le critique. La dimension sadienne s'affirme chez Beauvoir tandis que la dimension sadique revient à Réage. Sur le plan du corpus que nous avons retenu ensuite, il est à noter qu'Éric Marty ne mentionne pas Réage et qu'il interprète les textes de Beauvoir sous un angle philosophique et politique essentiellement. Or notre point de vue se situe ailleurs : nous voulons questionner la démarche de Beauvoir et son analyse de Sade à travers sa conception de la femme.

La relation qui unit Beauvoir à Sade est complexe ${ }^{9}$. Il est nécessaire de distinguer, dans Faut-il brûler Sade?, ce qui relève de l'énonciation et ce qui a trait à l'énoncé. Beauvoir déclare en introduction vouloir écrire un pamphlet contre les privilégiés dont Sade est l'hapax : «Il [Sade] a revendiqué sous sa forme la plus extrême l'arbitraire de son bon plaisir ${ }^{10}$ », mais au fil des pages elle se révèle subjuguée et analyse littérairement les romans

8. Éric Marty, op. cit., p. 23-24.

9. Pour une analyse complémentaire du regard beauvoirien sur Sade, voir l'article de JeanChristophe Abramovici, "Faut-il brûler Sade?" Rigueur de l'empathie », Cahiers de l'Herne, no 100, 2012, p. 316-319.

10. Simone de Beauvoir, Faut-il brûler Sade?, Paris, Gallimard, 1972, p. 7. 
du marquis. Ce qui la fascine chez Sade est sa capacité à sortir du dilemme entre soi et l'autre par la sexualité : «De sa sexualité il a fait une éthique, cette éthique il l'a manifestée dans une œuvre littéraire; c'est par ce mouvement réfléchi de sa vie d'adulte que Sade a conquis sa véritable originalité $^{11} \gg$. Beauvoir montre combien la sexualité imaginaire est une troisième voie entre isolisme et appétit sexuel débordant pour imaginer le bonheur par la hiérarchie. Sade transforme sa féodalité perdue en analogon littéraire sous la forme de la domination sexuelle. C'est cette transposition littéraire qui intéresse Beauvoir, bien plus que la simple volonté de puissance de l'aristocrate. Elle conclut en montrant l'apport ontologique de Sade : « Il nous oblige à remettre en question le problème essentiel qui sous d'autres figures hante ce temps : le vrai rapport de l'homme à l'homme ${ }^{12} \gg$. En revanche, si la sexualité illimitée est imaginaire, le désir masculin est bien réel. La lecture de Beauvoir est influencée sur ce point par Sartre, qui marque la violence du désir mâle lorsqu'il démontre que la liberté de la conscience incarnée d'autrui est précaire, le désir de pénétration mâle annihilant le caractère de sujet de l'autre pour en faire un pur objet ${ }^{13}$. La seule issue pour la liberté est donc individuelle. Pour Angela Carter, la mince possibilité d'émancipation de la femme sadienne consiste à participer de façon volontaire à la destruction : « Être une femme libre dans une société qui ne l'est pas, implique un comportement monstrueux ${ }^{14} »$. La femme n'a donc pour première solution que de prétendre agir à l'instar des hommes : « dans un siècle entièrement corrompu le plus sûr est de faire comme les autres ${ }^{15}$ ». Sade, et Beauvoir à sa suite, proposent ainsi des variantes à la dialectique hégélienne. Sade assure dans ses fictions un retournement possible au profit de l'esclave selon le bon vouloir du maître : c'est le cas de Juliette et des historiennes. Dans Le Deuxième Sexe, Beauvoir montre que la femme est pour l'homme le moyen d'échapper à la dialectique - c'est ce qu'elle décrit dans la section « Mythes » de son essai : la femme « est l'intermédiaire souhaité entre la nature étrangère à l'homme et le semblable qui lui est trop identique. [...] Grâce à elle, il y a un moyen d'échapper à l'implacable dialectique du maître et de l'esclave qui a sa source dans la réciprocité des libertés ${ }^{16}$ ». La seconde solution est la prostitution choisie. La figure de la prostituée représente l'indépendance; elle soumet l'homme à la chair sans possibilité de domination. Â ce propos, Beauvoir cite Sade :

11. Ibid., p. 15.

12. Ibid., p. 82.

13. Voir Jean-Paul Sartre, L'Être et le Néant [1943], Paris, Gallimard, 1965, p. 468 et suiv.

14. Angela Carter, La Femme sadienne, traduit de l'anglais par Françoise Cartano, Paris, Henri Veyrier, 1979, p. 50-51.

15. Sade, Les Infortunes de la vertu, Paris, Gallimard, coll. « Folio », 1970, p. 76. Sade place ironiquement ce propos au rang des sophismes philosophiques.

16. Simone de Beauvoir, Le Deuxième Sexe [1949], Paris, Gallimard, coll. « Folio », 1976, t. I, p. 239. 


\begin{abstract}
C'est sur des femmes que Sade et Sacher Masoch assouvissent les désirs qui les hantent; leurs disciples, et la plupart des hommes qui ont des « vices » à satisfaire, s'adressent le plus ordinairement aux prostituées. Elles sont de toutes les femmes celles qui sont le plus soumises au mâle, et qui cependant lui échappent davantage ${ }^{17}$.
\end{abstract}

Sade déconstruit donc le mythe en prônant un idéal scandaleux; Beauvoir en recommandant la femme et l'union libres. Or l'union libre convient à la femme uniquement si elle ne lui a pas été imposée par l'homme qui, selon Beauvoir, se plaît à posséder la femme et non une femme, tandis que l'exclusivité caractérise les femmes. Sade préfigure donc la femme moderne, mais toujours de façon ambivalente, puisqu'il est question d'agir dans la duplicité. La réversibilité est valorisée dans le monde sadien où toutes les personnes censées être vertueuses s'avèrent maléfiques; mais chez Beauvoir, ce jeu est plus problématique. Elle laisse entendre que le désir profond de la femme est de rester sous la domination de l'homme, lui qui est protection et virilité. C'est pourquoi la libération dans Le Deuxième Sexe est étrangement résolue in fine. C'est peut-être également la raison pour laquelle Juliette est toujours en état de dépendance; Noirceuil lui déclare : "Je veux que tu sois femme et esclave avec mes amis et moi, despote avec tous les autres ${ }^{18} »$. Par l'action, la femme échappe à son aliénation, mais c'est paradoxalement en adoptant le mode d'être de l'homme et son idéal. La femme occupe cette position en équilibre : elle est à la fois l'indispensable, tant pour le libertin que pour l'homme des années 1950, mais aussi l'autre qui risque d'être éliminé selon le bon vouloir de l'homme tout-puissant.

Réage exacerbe ces analyses, elle qui recourt à une écriture mettant en avant l'aspect sadique de la relation homme/femme. Les éléments qui ont pu fasciner Beauvoir, Réage les développe à l'extrême. En effet, elle est soumise à de multiples influences :

C'est une référence à Sade qui s'impose avec le plus d'évidence, toutefois pas simplement à l'œuvre de Sade proprement dite, mais aux multiples appréciations auxquelles elle a pu donner lieu en France, dans les milieux intellectuels, après la fin de la Deuxième Guerre mondiale ${ }^{19}$.

Le sadisme décrit dans Histoire d'O est donc particulièrement compliqué, mêlant servitude volontaire, fascination pour le bourreau et scènes pornographiques stéréotypées. L'on rencontre ainsi naturellement au sein du récit le cas le plus flagrant de retournement de la dialectique hégélienne : l'esclave veut rester esclave. La narration opère même un

17. Ibid., p. 315.

18. Sade, Histoire de Juliette, ou les Prospérités du vice, dans CEuvres complètes du marquis de Sade, Paris, Pauvert, t. VIII, 1987, p. 239.

19. Susan Sontag, L'Euvre parle, Paris, Seuil, 1968, p. 266. 
redoublement de l'esclavage : O est soumise à René qui est soumis à Sir Stephen. La relation maître/esclave y est nette :

Elle ne l'appelait jamais que Sir Stephen, comme une étrangère, ou comme une servante. Elle se disait que le mot «Seigneur » eût mieux convenu, si elle avait osé le prononcer, comme lui convenait à elle, en face de lui, le mot d'esclave ${ }^{20}$.

C'est l'acceptation de l'homme sadique qui donne son caractère scandaleux à Histoire d'O, bien plus que son caractère pornographique. L'œuvre de Sade a donc deux conséquences : la revendication de la libération de la femme pour Beauvoir, la soumission volontaire de la femme chez Réage. Pour Régine Deforges, les deux postures ne sont pas incompatibles : «On peut être féministe et prendre plaisir à être, comme $\mathrm{O}$, un objet sexuel. Car qui a décidé d'être cet objet, si ce n'est elle ${ }^{21}$ ? » L'esclavage volontaire - la préface de Paulhan au roman s'intitule « Le bonheur dans l'esclavage »- est paradoxalement libérateur pour O. La fin tronquée du roman, laissant ouvert l'avenir d'O, montre que cette dernière est prête à donner sa vie pour l'être aimé, dans un don total d'elle-même :

Dans un dernier chapitre, qui a été supprimé, O retournait à Roissy, où Sir Stephen l'abandonnait. Il existe une seconde fin à l'histoire d'O. C'est que, se voyant sur le point d'être quittée par Sir Stephen, elle préféra mourir. Il y consentit ${ }^{22}$.

Stylistiquement, le refus de représenter l'éternel féminin se traduit par l'usage de stéréotypes et la manifestation d'écarts. Le mythe féminin est relayé par des clichés, tandis que la narration prend en charge la distance critique. Les femmes apparaissent tels des archétypes de contes de fées chez Sade - «Justine se lit, ou se devrait lire, comme un conte de fées ${ }^{23}{ }^{23}-$ tout comme chez Réage - O l'aventurière, Anne-Marie la perverse - et les portraits physiques importent moins que la mise en scène des corps. Tout est facticité et sur-jeu chez Sade pour Beauvoir ${ }^{24}$. S'ensuit une forte abstraction des personnages, y compris chez Beauvoir qui abstrait la femme et dresse des stéréotypes tout au long du Deuxième Sexe. Sade et Réage sont dans l'écriture du fantasme, ce qui justifie cet usage des clichés du genre, comme l'exprime Réage en interview : «Les supplices et les violences dans Histoire d'O sont tout à fait du même ordre que les bagarres dans les romans policiers. [...]. C'est, si vous voulez, une espèce de convention du genre ${ }^{25} »$. La référence

20. Pauline Réage, op. cit., p. 124.

21. Régine Deforges, O m'a dit : entretiens avec Pauline Réage [1975], Paris, Pauvert, 1995, avant-propos, p. III.

22. Pauline Réage, op. cit., p. 201.

23. Jean Paulhan, « La douteuse Justine ou les revanches de la pudeur », préface aux Infortunes de la vertu, op. cit., p. 24.

24. Voir Simone de Beauvoir, Faut-il brûler Sade?, op. cit., p. 45 et suiv.

25. Régine Deforges, op. cit., p. 107-109. 
constante à Sade participe de cette convention; elle évite toute description précise, fonctionnant comme un signe transparent - Paulhan définit $\mathrm{O}$ dans la préface en la nommant Justine ${ }^{26}$. L'esprit de sérieux des œuvres les conduit alors à refuser les clichés sur l'amour en démétaphorisant les lieux communs amoureux. Le don de soi des mystiques et des amoureux est pris au sens propre par Sade : les femmes donnent entièrement leur corps à autrui. De même, Paulhan prend dans leur sens littéral les expressions amoureuses littéraires ou communes, permettant ainsi de justifier la cruauté exercée sur les femmes dans Histoire d'O en créant une filiation littéraire inattendue :

Il se peut, après tout, que la tunique ardente des mythologies ne soit pas simple allégorie; ni la prostitution sacrée, curiosité de l'histoire. Il se peut que les chaînes des chansons naïves et les « je t'aime à en mourir » ne soient pas une simple métaphore. Ni ce que disent les rôdeuses à leur amant de cœur : « Je t'ai dans la peau, fais de moi ce que tu voudras $»^{27}$.

Histoire d'O, dans la filiation de Sade, serait le récit éprouvant les lieux communs sur l'amour.

\section{Féminin et masculin. La revanche de Juliette en question}

Ces paradoxes de la littérature érotique informent la vision de Beauvoir. On observe dans ses travaux un phénomène d'inversion, de retournement constant. Beauvoir dans Privilèges désire en introduction critiquer Sade; or elle le met en valeur in fine. Dans Le Deuxième Sexe, déjà, elle critiquait la domination de l'homme mais le deuxième tome se clôt étrangement sur une forme de statu quo :

Affranchir la femme, c'est refuser de l'enfermer dans les rapports qu'elle soutient avec l'homme, mais non les nier; qu'elle se pose pour soi elle n'en continuera pas moins à exister aussi pour lui : se reconnaissant mutuellement comme sujet chacun demeurera cependant pour l'autre un autre ${ }^{28}$.

L'enfermement de la femme dans le statut d'« autre » n'est finalement pas dépassé par Beauvoir dans le sens où il n'y a pas de retournement hiérarchique au profit du pôle féminin. L'égalité est instaurée, la femme n'a pas de prérogatives. C'est ce que reprochera à Beauvoir une frange des féministes des années 1960, regrettant l'instauration d'une égalité sans la différence ${ }^{29}$. Ces phénomènes d'inversion se retrouvent ailleurs : pour Paulhan, la femme est paradoxalement le personnage important de Sade ${ }^{30}$;

26. Pauline Réage, op. cit., p. 10-11.

27. Jean Paulhan, «Le Bonheur dans l'esclavage », préface à Histoire d'O, Paris, Pauvert, 1975, p. 16-17.

28. Simone de Beauvoir, Le Deuxième Sexe, op. cit., t. II, p. 652.

29. Sylvie Chaperon, «Beauvoir et le féminisme français », Cahiers de l'Herne, no 100 , 2012, p. 277-283, p. 280.

30. Jean Paulhan, « Justine, c'est lui » (dans « La douteuse Justine », op. cit., p. 51). 
chez Réage, la domination de la femme s'accomplit dans l'esclavage. Ces renversements expliquent alors pourquoi Beauvoir ne critique pas Sade dans son essai : il n'y a pas une totale soumission des femmes dans Sade, mais un basculement possible. Juliette le symbolise explicitement, la résistante et vertueuse Justine implicitement, elle dont il faudrait réinterroger le «masochisme de la faiblesse et du demi-consentement ${ }^{31}$ ». Il faudrait, pour que la femme soit pleinement libre, trouver une voie entre la femme esclave, l'épouse soumise et Diane, l'amazone... C'est toujours le problème de l'identité féminine qui ressurgit dans la littérature érotique et féministe : la femme doit être successivement, voire simultanément mère, amante et épouse. Ne pouvant revêtir toutes ces facettes, la femme est désignée comme l'inférieure à un moment ou un autre du récit. La femme pour exister de façon autonome doit être plurielle, telle est la leçon de Sade à travers le catalogue de femmes que dressent ses romans : Justine, Aline... La femme idéale sera celle qui mêle l'ensemble des caractéristiques des personnages décrits dans les différents romans de Sade.

À partir de cette vision ambivalente, il est possible de réinterroger la question de la domination masculine. Les œuvres de Sade illustrent les fantasmes masculins sur les femmes, comme l'a noté Angela Carter. Mais deux siècles plus tard, l'instigatrice du féminisme n'échappe pas non plus à ces représentations phallo-centrées. Et Beauvoir conserve un point de vue masculin sur les femmes :

On pourrait sans grand paradoxe soutenir que Le Deuxième Sexe, réputé « livre sur les femmes », est en réalité un livre sur les hommes. [...] Cet arrangement, qui reflète la traditionnelle assimilation de l'homme à la structure qui encadre et la femme à la chair modelée, pourrait déjà préjuger du résultat de l'investigation qui est le sujet du livre, sur un état de fait donné comme énigmatique et pourtant ayant toujours déjà existé, la domination des hommes sur les femmes que désigne la contrainte du livre ${ }^{32}$.

Le masculin domine toujours, et Beauvoir renverse le but qu'elle s'était donné, c'est-à-dire libérer la femme : "Son féminisme, compliqué de revendication existentialiste, s'occupe surtout, en fin de compte, à dire que l'on n'est pas femme, à refuser la féminité ${ }^{33} \gg$. Son œuvre fictionnelle postérieure, Les Mandarins, ne met en scène aucun couple harmonieux et les femmes y sont encore largement dominées par les hommes, y compris

31. Béatrice Didier, introduction aux Infortunes de la vertu intitulée « La probable Justine ou les secrètes revanches du libertinage », Paris, Gallimard, 1970, rééd., coll. " Folio classique », p. 63.

32. Annette Lavers, «Le mythe de la femme dans la vie quotidienne », dans Ingrid Galster (dir.), Simone de Beauvoir : Le Deuxième Sexe. Le livre fondateur du féminisme moderne en situation, Paris, Champion, 2004, p. 261.

33. Armand Hoog, "Madame de Beauvoir et son sexe », La Nef, n 56, août 1949 (dans la chronique «Les Lettres françaises »), dans Ingrid Galster (dir.), Le Deuxième Sexe de Simone de Beauvoir, Paris, Presses de l’Université Paris-Sorbonne, 2004, p. 164. 
dans l'amour adultère - domination de Lewis Brogan sur Anne. Le vœu de Carter d'une pornographie au service de la femme s'avère un vœu pieux. Carter marque le même échec de la féminité chez Réage; le récit qui donne à entendre les sentiments d'O ne change rien au fait que ce texte pornographique est écrit à l'adresse des hommes. La femme sadienne des années 1950 reste un objet, même si elle est narratrice. La domination narrative est restreinte, car le jouisseur est toujours déjà désigné, et c'est l'homme. Sade comme Beauvoir en restent ainsi au stade de la rébellion, de la libération désirée de la femme, sans atteindre le stade de la liberté au long cours. Beauvoir ne dénonce pas suffisamment la violence faite aux femmes; elle s'installe dans la polémique mais ne propose aucune solution pérenne. Cette position sur le fil conduit à un contre-emploi de la démonstration :

Les féministes, et singulièrement Mme Simone de Beauvoir, ne sont pas celles qui veulent se libérer du sexe masculin, mais plutôt celles qui veulent se libérer du sexe de l'homme et de leur propre sexe. Leur désir de libération est avant tout un étrange et insupportable sentiment de servitude ${ }^{34}$.

Le personnage de la Delbène, dans l'Histoire de Juliette, qui donne à Juliette les bases d'une approche " féministe ", n'a finalement pas de descendante au $\mathrm{Xx}^{\mathrm{e}}$ siècle. Sade se situe dans l'interstice entre la libération voulue de la femme et son impossible liberté, c'est-à-dire entre la vision de Beauvoir et celle de Sartre, qui démontre, comme nous l'avons vu supra, que le désir se meut en sadisme. Pour Sartre, il n'existe pas de réciprocité entre le masculin et le féminin; son regard machiste - qui est aussi un regard d'époque, ce que Judith Butler nomme la « culture masculiniste $^{35} \gg-$ agit largement sur la position beauvoirienne ${ }^{36}$, et l'analyse sartrienne du sadisme empêche toute compréhension complexe de l'œuvre du marquis :

Le sadique ne peut, en effet, rendre présente à autrui son incarnation que sous la forme de l'obscène, qui appartient, souligne Sartre, au genre du disgracieux. Alors que dans la grâce, la liberté contient la facticité, le sadique vise à faire qu'en l'autre la facticité déborde la liberté ${ }^{37}$.

Or Juliette incarne cette liberté échappant à l'emprise du regard libertin.

Afin de dépasser cette binarité multiséculaire du masculin et du féminin, nos auteurs envisagent les représentations d'une troisième voie.

34. Ibid., p. 162.

35. Judith Butler, Trouble dans le genre, op. cit., p. 51.

36. Pour les influences réciproques entre Sartre et Beauvoir, voir notamment Catherine Poisson, Sartre et Beauvoir : du je au nous, Amsterdam, Rodopi, 2002.

37. Michel Kail, «Le masculin et le féminin. Sartre et Beauvoir regards croisés », Sens public, [En ligne], http://www.sens-public.org/article.php3?id_article=427, mis en ligne en avril 2007, consulté le 16 août 2012. 
Sade crée des personnages qui sont à la frontière de l'animalité, et qui frôlent la catégorie du monstrueux par leurs appétits inassouvis. Il campe des personnages à l'identité sexuelle floue, oscillant entre hétérosexualité et homosexualité. L'acmé de cette question du genre est atteinte à la fin de La Philosophie dans le boudoir (1795), avec la scène de Mme de Mistival aux orifices cousus par sa propre fille Eugénie ${ }^{38}$, qui aboutit en fait à une créature qui n'existe pas. Ce personnage monstrueux n'est pas une mère - « Maman, que je vous couse, afin que vous ne me donniez plus ni frères ni sœurs ${ }^{39} \gg-$, n'est pas une femme - elle n'a plus les moyens d'avoir des relations sexuelles -, mais n'est pas non plus un homme - aucun organe sexuel mâle; sorte d'être asexué, d'objet inanimé, d'être neutre abject comme l'analyse Barthes. Cette revendication d'un neutre générique ressurgit selon d'autres modalités dans les années 1950, chez nos deux auteurs. Pour Beauvoir, le neutre est accaparé par l'homme du fait de l'étymologie : le sens singulier de «vir » s'est assimilé au sens général du terme « homo ». L'homme est le masculin et l'humain par excellence. Beauvoir cherche alors en contrepartie à faire de la femme, qui est toujours l'Autre, une personne qui ne soit ni mère, ni épouse, ni soumise ni totalement féministe. Cet état de femme non féminine est atteint à l'âge de la ménopause; Beauvoir parle alors de « troisième sexe ${ }^{40}$ ", car la femme n'est plus soumise à l'espèce, sans être un mâle pour autant. En dehors de cette évolution biologique, Beauvoir vise à dé-générer le genre. On peut alors relire son analyse de la biologie et de l'hermaphrodite ainsi que son chapitre sur la figure de la lesbienne, dans le second tome, comme des revendications fortes d'entités doubles qui la fascinent. L'homosexualité est « pour la femme une manière parmi d'autres de résoudre les problèmes posés par sa condition en général, par sa situation érotique en particulier ${ }^{41} \gg$. Beauvoir réfère au mythe platonicien de l'androgyne comme moment de fusion parfaite. Les revendications de Beauvoir anticipent en quelque sorte la définition du neutre par Barthes dans les années 1970 , comme refus de penser le genre ${ }^{42}$. Cette position de Beauvoir n'est pas admise dans les années 1950; ainsi Aimé Patri refuse cette dé-génération :

38. Annie Le Brun voit en Eugénie un double de Juliette : «Si Juliette a une sœur qui lui ressemble, c'est Eugénie de Mistival, et non pas Justine » («Avant et après Juliette », préface à Sade, Histoire de Juliette, op. cit., p. 34).

39. Sade, La Philosophie dans le boudoir, Paris, 10/18, 1972, p. 249.

40. Simone de Beauvoir, Le Deuxième Sexe, op. cit., t. I, p. 69.

41. Simone de Beauvoir, Le Deuxième Sexe, op. cit., t. II, p. 215. Nous soulignons.

42. «Un premier état de $S / Z$, paru dans un hommage à Jakobson, s'intitulait significativement : "Masculin, féminin, neutre." Le neutre est très exactement le ne-uter, ce qui est ni l'un ni l'autre (sexe). La fascination, pour Barthes, du castrat balzacien tient au fait qu'il n'est, dans la nouvelle, ni une femme imaginaire (homme travesti) ni un homme réel (inverti), mais qu'il est cet innommable du discours, ce hors catégorie, ce rien qui le propulse hors sexe - et hors sens, puisque le sens s'articule au phallus » (Serge Doubrovsky, Parcours critique II (1959-1991), texte établi par Isabelle Grell, Grenoble, Ellug, 2006, p. 69). 
Si, par égalité, on entend interchangeabilité, il faudrait que, les hommes ayant déjà fait couper leurs barbes et les femmes leurs cheveux, on assiste à l'avènement d'une espèce de «neutres » comparables aux « ouvrières » des ruches d'abeille qui sont, comme on le sait, des femelles dégénérées en un certain sens. [...]. Simone de Beauvoir s'est faite le porte-parole des femmes en révolte contre leur condition et c'est ce qui fait la signification de son livre. Mais, bien que la formule de « l'égalité de la différence » offre à ses oreilles une résonance " raciste ", j'espère avoir suggéré que, pour s'acheminer vers une solution des problèmes du « dimorphisme », il est une autre voie que celle de l'« amorphisme ${ }^{43}$.

Histoire d'O se clôt sur l'image du personnage éponyme déguisé en chouette; par cette animalisation, l'anéantissement des caractéristiques féminines touche à son but. Parallèlement à cela, dans la préface, Jean Paulhan parle d'une femme virile pour qualifier l'auteure et $\mathrm{O}^{44}$.

Un autre contrepoint au couple masculin/féminin se lit dans Histoire d'O, qui rejoint la figure beauvoirienne de la lesbienne : le gynécée. Le gynécée formé par Anne-Marie représente un idéal de vie et d'amour pour O, balançant l'épisode masculin du château de Roissy. Si l'on suit l'interprétation de Monique Wittig sur la figure de la lesbienne, le personnage d'Anne-Marie incarne la troisième voie :

Une lesbienne transcende [...] l'opposition binaire entre la femme et l'homme; une lesbienne n'est ni une femme ni un homme [...] une lesbienne n'a en fait pas de sexe; elle se situe au-delà des catégories de sexe. [...] la lesbienne semble être un troisième genre [...], une catégorie qui rend le sexe et le genre tout à fait problématiques en tant que catégories politiques stables de description $^{45}$.

De façon contemporaine, Judith Butler refuse le pouvoir masculin qui s'exerce en vérité et dans les discours, et reprend la quête de Beauvoir; « la tâche de cette réflexion est de se dé-centrer - et de déstabiliser - de telles instances de définition : le phallogocentrisme et l'hétérosexualité obligatoire $^{46} \gg$. Elle reprend le raisonnement de Beauvoir là où celle-ci s'est arrêtée. En effet, Judith Butler accepte la fameuse phrase de Beauvoir «On ne naît pas femme : on le devient » : pour elle, Beauvoir distingue raisonnablement le sexe immuable et le genre qui relève de l'acquis. Mais dans les années 1990, Judith Butler est en mesure d'aller plus loin :

S'il est impossible de changer de sexe - c'est du moins ce qu'elle pensait - le genre est, quant à lui, la construction culturelle et variable du

43. Aimé Patri, «Y a-t-il un éternel féminin?», Paru, août-septembre 1949, cité dans Ingrid Galster (dir.), Le Deuxième Sexe de Simone de Beauvoir, op. cit., p. 184-185.

44. Jean Paulhan, «Le Bonheur dans l'esclavage », op. cit., p. 10-11 : «Femme il se peut, mais qui tient du chevalier, et du croisé ».

45. Cité dans Judith Butler, Trouble dans le genre, op. cit., p. 225.

46. Ibid., p. 53. 
sexe $[\ldots]$. La théorie de Beauvoir a des conséquences, semble-t-il, radicales qu'elle même n'arrivait pas à imaginer ${ }^{47}$.

Si le sexe ne limite pas le genre ${ }^{48}$, il y a alors plusieurs genres possibles, et non seulement deux. Judith Butler s'intéresse alors à une figure transgenre, celle de la drag-queen, qui semble clore provisoirement notre réflexion en instituant le " trouble dans le genre » : la figure du drag mêle le refus du genre que l'on a observé chez nos trois auteurs, l'importance du spectacle et de la mise en scène, si capitale chez Sade, et la notion de parodie, qui touche aux clichés des récits pornographiques. Cette figure complexifie aussi la question du féminisme, dont Wittig semblait trouver la résolution absolue dans le lesbianisme ${ }^{49}$. Notons un dernier point : Judith Butler revendique un engagement éthique fort pour le respect des minorités sexuelles, elle prolonge et rend décisives les revendications de Beauvoir. Les liens de Beauvoir avec le mouvement féministe sont complexes et en tout cas distants dans les années que nous explorons ici ${ }^{50}$. Gisèle Halimi remarque au sujet de la concrétude du combat de Beauvoir :

Dans Le Deuxième Sexe, elle n'envisageait pas de stratégie des luttes des femmes et concluait que le socialisme suffirait pour que tous les problèmes des femmes soient résolus. Pour elle, le combat féministe n'était pas un combat spécifique ${ }^{51}$.

Pourrions-nous conclure par une équation à trois éléments connus? L'addition de Sade et du Deuxième Sexe donnerait-elle pour résultat Histoire d'O? Il paraît en réalité que la terreur masculine, telle qu'elle est pratiquée dans les romans de Sade, ne soit pas totalement annulée par l'essai féministe de Beauvoir. Histoire d'O est un roman qui mêle terreur et douceur, sans aboutir à une libération nette de la femme, et encore moins à une supériorité

\section{Ibid., p. 223.}

48. Non seulement le sexe ne limite pas le genre, mais le sexe, dans sa matérialité, est influencé par nos représentations culturelles selon Judith Butler (c'est aussi la représentation qu'en a Sade, lui qui propose dans ses romans une remise en question des normes sexuées et de la préhension même des corps). Le sexe est normé, tout comme le genre. Beauvoir n'interrogeait pas encore la matérialité du sexe, sa théorie s'appuyant sur des différences sexuelles d'ordre corporel. Voir Judith Butler, Ces corps qui comptent. De la matérialité et des limites discursives $d u$ « sexe » [Bodies that matter, 1993], trad. Charlotte Nordmann, Paris, Amsterdam, 2009.

49. Judith Butler, Trouble dans le genre, op. cit., p. 246.

50. Michel Kail explore en partie la question du lien de Beauvoir au féminisme dans son article «De la féminité à l'humanité », Cahiers de l'Herne, nº 100, 2012, p. 271-276, notamment, p. 275-276. Voir également l'article de Sylvie Chaperon, «Beauvoir et le féminisme français », op. cit., note 28, p. 65 .

51. Gisèle Halimi, propos recueillis par J. Bonnier-Hamon, S. Dupagny et V. Lucas, Choisir, $\mathrm{n}^{\circ} 102$, février 2008, repris dans « La figure féministe la plus importante », Cahiers de l'Herne, no 100, 2012, p. 303. 
de la « classe des femmes » - au sens où Nicole-Claude Mathieu ${ }^{52}$ définit cette expression en montrant que les mouvements féministes et lesbiens défendent cette idée de classe en opposition à la classe dominante, celle des hommes.

Deux ans après la publication d'Histoire d'O, le texte de Catherine Robbe-Grillet, masquée derrière le pseudonyme de Jean de Berg, L'Image ${ }^{53}$, donne alors une version plus positive du couple, au sein d'un univers pornographique. Elle peint des femmes de talent, qui piègent la gent masculine, mais non par pur sadisme. L'Image représente alors le verso d'Histoire d'O, texte scandaleux par sa paradoxale résolution romantique :

Le genre de la pornographie littéraire comporte une branche qui s'apparenterait à la tragédie (comme dans Histoire d'O) où le sujet subissant l'expérience érotique s'achemine inexorablement vers la mort, et une autre, proche de la comédie (comme dans L'Image) où la poursuite obsessionnelle de l'expérience sexuelle se termine d'une façon heureuse par l'union avec un partenaire, seul capable d'apporter sur ce plan les satisfactions désirées ${ }^{54}$.

Pour le lectorat féminin, cet autre texte des années 1950 donne un contrepoint aux héritières mortifères de Sade.

Perrine Coudurier Université Paris-Sorbonne, Littérature française XIXe-XXI siècles

52. Voir notamment Nicole Claude-Mathieu, L'Anatomie politique. Catégorisations et idéologies du sexe, Paris, Côté-femme, 1991.

53. Jean de Berg, L'Image, Paris, Minuit, 1956.

54. Susan Sontag, op. cit., p. 276-277. 